\title{
Teoría de la complejidad y clúster industrial: caso de un clúster de software
}

Flores Sánchez Carlos Alberto

carlos.flores@uabc.edu.mx

https://orcid.org/0000-0003-1516-166X

Universidad Autónoma de Baja California

Osuna Millán Nora del Carmen

nora.osuna@uabc.edu.mx

https://orcid.org/0000-0001-5719-7682

Universidad Autónoma de Baja California

Ricardo Fernando Rosales Cisneros
ricardorosales@uabc.edu.mx
https://orcid.org/0000-0002-0266-2951
Universidad Autónoma de Baja California

Recepción: 15/09/2021

Aceptación: 18/10/2021

\section{Resumen}

Tanto el concepto de clúster industrial como el de teoría de la complejidad no son temas nuevos, sin embargo, ambos han emergido en la literatura de los últimos 20 años como una forma diferente de organización, en el caso del clúster, y de enfoque en el caso de la teoría de la complejidad. El presente documento busca introducir la teoría de la complejidad como instrumento de análisis de clústeres industriales. Para esto se usa la metodología de caso de estudio sobre la experiencia en la construcción de un clúster de tecnologías de información y comunicación. Los resultados del trabajo muestran como las características de la teoría de la complejidad tienen fuertes similitudes con la dinámica de los clústeres, lo que ayuda al entendimiento de estas dinámicas. Se concluye que la teoría de complejidad es una herramienta, que conforme las dinámicas de mercado y avance tecnológico van sucediendo, se vuelve más fundamental para la comprensión y el apoyo al desarrollo de estas aglomeraciones.

Palabras clave: Clúster, teoría de la complejidad, clúster de software

\section{Abstract}

Both the concept of industrial cluster and complexity theory are not new topics; however, both have emerged in the literature of the last 20 years as a different form of organization, in the case of the cluster, and focus on the case of complexity theory. This document seeks to introduce complexity theory as an instrument for analyzing industrial clusters. For this, the case study methodology is used on the experience in the construction of a cluster of information and communication technologies. The work results show how the characteristics of complexity theory have strong similarities with the dynamics of clusters, which helps to understand these dynamics. It is concluded that complexity theory is a tool that becomes more fundamental for understanding and supporting the development of these agglomerations as the market dynamics and technological advances take place. 
Keywords: Cluster, Complexity theory, cluster software

\section{Introducción}

Ni la teoría de la complejidad, ni la literatura de clústeres son temas nuevos, sin embargo, su aplicación y enfoque en las dinámicas organizacionales tiene poco mas de 20 años. La industria de las TIC's, en la ciudad de Tijuana, ha manifestado una fuerte dinámica principalmente a principio del siglo XXI, esto propiciado por una fuerte demanda de infraestructura de tecnologías de información por parte de Estados Unidos de Norteamérica. Siendo California uno de los principales estados en cuanto a economía y que colinda con el Estado de Baja California, México.

No es extraño que en muchas industrias exista una dinámica de complementariedades entre esta zona fronteriza, donde California ve a la Baja California como una oportunidad de abaratar costos, desde insumos hasta mano de obra. Por otro lado, Baja California también ve como oportunidad al mercado Californiano con necesidades de insumos que cumpla sus expectativas y de mano de obra preparada (bien pagada en California). En esta dinámica es que en los 90 inician en la región el desarrollo de programas académicos para satisfacer esta demanda. Así como, el surgimiento de empresas que buscan satisfacer esa necesidad principalmente de soporte técnico y de desarrollo de software. Bajo este contexto es que, a principios del siglo XXI, inicia una dinámica empresarial, en búsqueda de un mejor aprovechamiento de la situación de mercado que se hablo anteriormente. Esta iniciativa surge de experiencias observables y documentadas como el caso del Silicon Valley (Norberg, 1992), de la Route 128 (Rosegrant y Lampe, 1992), del Bangalore Cluster TIC's (Basant, 2006), y de otras experiencias en México por ejemplo en Mérida, Yucatán, el caso del Centro de la Industria de la Tecnología de Información (CITI), en Monterrey el Consejo de Software de Nuevo León, en Zapopan, Jalisco, el Instituto Jalisciense de Tecnologías de Información (IJALTI).

Estas experiencias documentadas que hablan sobre la dinámica empresarial que logra nuevas formas de organización, donde predomina una relación de Triple Hélice (Ramírez et al, 2012). Donde se busca centrar el trabajo en la cooperación para lograr la innovación, la productividad y la competitividad, donde el aprendizaje y el conocimiento sirven para diversificar los mercados y hacer frente a la competencia y aprovechar los avances tecnológicos.

En este documento primeramente se desarrollan las características de la teoría de la complejidad, para después conceptualizar los clústeres industriales, se desarrolla la metodología, se muestran los resultados del análisis de aso práctico bajo en enfoque de la teoría de la complejidad y por último se muestran las conclusiones.

\section{Características de la teoría de la complejidad}

Un sistema complejo se puede definir como un sistema compuesto por muchas partes interconectada que interactúan de una forma no lineal, y que es capaz de mostrar propiedades de organización y surgimiento. A continuación, se muestran las partes de un sistema complejo (Cilliers, 1998)

1. Agentes: los sistemas complejos están formados por una gran cantidad de agentes.

2. Interconexión: los agentes están interconectados y forman una red de vínculos

3. Conectividad: las interacciones entre agentes definen tres tipos de regímenes: la conectividad tiene lugar cuando todos los agentes están interconectados; es escasamente conectado cuando las redes cuentan con pocos canales de interacción; conectividad media, se describen como sistemas al borde del caos o en un estado de auto criticidad (Bak y Chen, 1991) (Waldrop, 1995).

4. No linealidad: las interacciones son comúnmente no lineales. Existen dos aspectos para la no linealidad: a) relación causa-efecto: no hay proporcionalidad directa entre entrada y salida y $b$ ) principio de supersición: los comportamientos de las partes individuales de los sistemas no pueden resumirse para producir un estado final.

5. Ciclo de retroalimentación: incluso en una red escasamente conectada, las interacciones retroalimentan creando un ciclo. El efecto de un ciclo puede ser un refuerzo mutuo entra causas y efecto (retroalimentación positiva) como en una reacción en cadena, y por lo tanto desestabilizante, o atenuación mutua (retroalimentación negativa) y por lo tanto estabilizador.

6. Sistemas complejos son sistemas disipativos: necesitan un flujo constante de energía de su entorno para mantener su grado interno de complejidad. Nicolisy Prigogine en 1989, distinguen entre equilibrio cercano y lejano de los sistemas disipativos de equilibrio: el primero se aproxima a un sistema cerrado en equilibro estable y que puede describirse mediante un enfoque lineal y reduccionista, que este ultimo esta abierto y tiene hambre de energía (o información).

7. Los sistemas complejos son sistemas evolucionarios: los sistemas complejos son evolutivos, es decir tienen una historia. No hay leyes generales que apliquen sobre las circunstancias de la evolución. Esto significa que la suerte a menudo juega un papel importante. Los agentes de un sistema comple- 
jo evolutivo no se puede homologar su comportamiento mediante un promedio.

8. Principio de localidad: los agentes obedecen al principio de localidad, que significa que reaccionan a la información y desconocen el contexto macro. Los agentes pueden ir más allá de su alcance del principio de localidad e internalizan parte de la complejidad en su red. Por otro lado, la re-flexibilidad se refiere a dar forma estratégica a su entorno adaptando una perspectiva crítica sobre ellos.

9. Auto organización: los sistemas complejos muestran una tendencia a auto estructurar sus patrones colectivos de comportamiento. Por ejemplo, en el caso de una bandada de pájaros volando en forma de flecha no siguen un líder o un conjunto genético o cultural predefinidos de instrucciones. Si no, la forma final es el macro comportamiento resultante de micro comportamientos. La auto organización indica la capacidad de un sistema para desarrollar o cambiar la estructura interna de forma espontánea y adaptativa para hacer frente a, o manipular su entorno (Cilliers, 1998). La auto organización no es precisamente resultado de una estrategia deliberada o de una necesidad genética, sino es resultado del nivel de coherencia que tiene lugar entre los actores individuales en la búsqueda de sus metas locales

10. Coevolución: por el papel fundamental de la conectividad, cualquier acción dentro del sistema es inherentemente de naturaleza circular. Las acciones no suceden de forma aislada, pero si tienen el potencial de desencadenar una serie de efectos sobre los nodos (agentes) con los que el nodo está interconectado. El efecto resultante es el lograr un efecto no aislado, la transformación de cualquier parte sin que se produzca un proceso simultáneo de adaptación en el resto del sistema. La interdependencia genera coevolución, que Kauffman (1995) describe como el proceso evolutivo donde cada paso dado por cualquier agente modifica el entorno de los demás.

11. Surgimiento: cuando los átomos se organizan en moléculas, dichas moléculas son los ladrillos de la vida. La auto organización genera una jerarquía de un mundo de sistemas complejos organizados y anidados, parcialmente desacoplados. El surgimiento de un sistema dentro de otro requiere de un aumento de orden en el nivel espaciotemporal de un nuevo sistema. Este proceso requiere energía porque invierte la tendencia de los sistemas a aumentar su desorden interno, medido por el concepto termodinámico de entropía. Este enfoque tiene consecuencias radicales: en primer lugar, si el surgimiento requiere de un orden y de un flujo negativo de entropía para apoyar el nuevo orden, entonces los sistemas en el equilibro son sistemas muertos y los sistemas activos deben ser disipativos (característica 6). En segundo lugar, donde las disciplinas de base newtoniana estudian sistemas en equilibrio como sinónimo de sistemas optimizados y estables, entonces la búsqueda de ciencias basadas en la complejidad por las leyes de los sistemas disipativos autoorganizados dinámicamente. Y, por último, siendo los sistemas newtonianos deterministas, una trayectoria definida vinculará la evolución de un sistema desde un conjunto de condiciones iniciales hasta el estado de equilibrio final, mientras que, de acuerdo con la complejidad, múltiples estados alejados del equilibrio podrían corresponder a el mismo conjunto de condiciones iniciales.

12. Reacciones auto catalíticas: un mecanismo que podría explicar la aparición de niveles jerárquicos ordenados es introducido por Kauffman (1995) sugiriendo que un conjunto de reacciones químicas se vuelve auto catalítico en presencia de una cantidad suficiente de diferentes moléculas. Si la variedad es suficientemente grande, entonces hay una alta probabilidad de que algunas moléculas tengan el doble papel de entrada / reactivo y producto de la reacción. Lo cual lleva a una mayor variedad y aceleración de la reacción. La auto catálisis genera un mecanismo de retroalimentación positiva que, por un lado, genera nuevas moléculas, lo cual se traduce en un aumento de la variedad interna del sistema. Por otro lado, conectando las moléculas existentes en una red de múltiples reacciones, se refuerza el proceso auto catalítico. Los modelos de masa crítica son útiles aquí (Shelling, 1978). Cuando se alcanza un punto crítico de algunas variables de control entonces el sistema puede volverse inestable y surge un conjunto ramificado de soluciones. Esto representa un punto de bifurcación (Nicolis y Prigogine, 1989), donde son las condiciones locales las que forzan al sistema a optar por una de las ramas de la bifurcación.

\section{Clusters}

El fenómeno de la aglomeración industrial tiene más de cien años como objeto de estudio de la actividad económica y de sus mecanismos. La aglomeración ocurre a diferentes escalas geográficas, desde áreas dentro de las ciudades hasta áreas entre continentes, así como a través de diferentes industrias, ya sea en actividades altamente especializadas o de la actividad económica a través de varias industrias (Lindqvist, 2009).

Alfred Marshall (1920) observó la concentración de fabricantes de sillas en Buckinghamshire y del comercio de cubiertos de mesa en Sheffield. Antes de esta obser- 
vación, se tenían ubicados lugares con fortalezas económicas como el caso de Ginebra en la industria de los relojes y el caso de Venecia como constructores de barcos. La historia ha mostrado que algunas aglomeraciones han sido persistentes, como el caso de Ámsterdam como centro editorial desde mediados del siglo XVII.

La aglomeración no afecta de la misma forma a todas las actividades económicas. Algunas son fuertemente concentradas, como el caso de los servicios financieros que suelen estar en los distritos financieros, mientras otras tienden a ser más dispersas como es el caso de las estéticas (salón de belleza). Sin embargo, con el fenómeno de la aglomeración surgen preguntas como ¿por qué existe?, ¿Qué factores y fuerzas atraen esas concentraciones?, y ¿Qué es lo que los mantiene unidos? A partir de estas preguntas han surgido varias teorías para explicar la aglomeración y por qué ocurre tal fenómeno espacial.

Alfred Marshall fue el primero en explicar el mecanismo de la aglomeración industrial, específicamente de una industria. En su trabajo sugiere que la ubicación de recursos para los negocios podría producir efectos de proximidad que denominó externalidades económicas (Marshall, 1920), de las cuales destaca cuatro: a) transferencia de habilidades e invenciones entre colegas, competidores y generaciones, b) surgimiento o crecimiento de industrias proveedoras a la industria central, con especialización en insumos y servicios, c) ventajas de escala en la compartición de maquinaria especializada, y d) mano de obra especializada.

Por su parte (Jacobs, 1969) propuso que la proximidad de varios negocios en actividades de diferentes campos, da lugar al surgimiento de nuevos tipos de negocio, y esto a su vez trae el crecimiento económico en las ciudades. De ambos autores se puede destacar que si las aglomeraciones existen, entonces deben existir efectos de proximidad dependientes, los cuales sirven para lograr su crecimiento y mantenerse (Lindqvist, 2009).

Entre 1940 y 1970 la geografía económica estuvo ausente en las corrientes principales de la teoría económica. Krugman (1995) atribuye el abandono al cambio a un riguroso modelado matemático en el análisis económico. Hasta el planteamiento del modelo de Dixit-Stiglitz de competencia monopolística, era prácticamente imposible incorporar economías de escala, de tal forma que las externalidades económicas eran simplemente ignoradas (Lindqvist, 2009). Sin embargo, entre 1980 y 1990, el fenómeno de las aglomeraciones volvió a llamar la atención de los investigadores de la nueva geografía económica y la nueva teoría de comercio, a partir de nuevos modelos para conceptualizar diferentes formas en que las economías de escala podrían provocar un crecimiento al comercio tanto internacional como interregional con un enfoque en la transferencia de conocimiento (Malmberg et al., 1996).
El enfoque en la aglomeración de industrias individuales o la integración de redes de compradores-proveedores, fue objeto de estudio en el área norte de Italia (Pyke et al., 1990). A este tipo de aglomeración la denominaron distrito industrial, el cual se caracterizó por una alta concentración geográfica de empresas en una pequeña área, con un perfil de especialización muy estrecho entre ellas. El predominio de las pequeñas y medianas empresas incrustadas en su ambiente local, permitió definir los distritos industriales como una entidad socio-territorial caracterizada por la presencia activa de una comunidad de personas y una población de empresas de una forma natural e histórica en un área determinada (Becattini, 1990). La especialización flexible y la producción en pequeños lotes de las pequeñas empresas se ven como una alternativa post-fordista a la producción a escala, caracterizada por corporaciones verticalmente integradas que dominaron las economías del oeste después de la segunda guerra mundial (Piore y Sabel, 1984).

La aglomeración de industrias en general se observa en el trabajo de Jacobs (1969), donde concluye que todas las innovaciones ocurren en las ciudades donde hay grandes cantidades de personas y empresas que representan diferentes tipos de conocimiento. Las ciudades permiten la división de trabajo y cuando este es combinado en nuevas formas ocurre la innovación, la cual mejora de manera continua por la atracción de una multitud de actividades en áreas cercanas. Una perspectiva similar enfatiza en la importancia de la clase creativa compuesta por personas que encuentran nuevas formas de realizar su trabajo (Florida, 2005). Esta clase creativa constituye aproximadamente un $30 \%$ de la fuerza de trabajo de Estados Unidos y migran gradualmente a centros creativos atraídos por las condiciones de vida que incluyen experiencias de alto nivel y apertura a la diversidad.

\section{Metodología}

La metodología que se utiliza es de un caso de estudio, donde se analiza la construcción del clúster de software de Tijuana conocido como BIT Center (Baja's Innovation and Technology Center). Se usa la teoría de la complejidad para explicar la dinámica que se dio en la construcción de dicho centro. Los casos de estudio son un modelo útil de investigación, que ayudan a estructurar la obtención de información en contextos de la vida real. Se utilizó esta metodología por que permite estudiar la dinámica de clúster desde la perspectiva de teoría de complejidad, así como explorar y obtener un conocimiento mas amplio del fenómeno clúster (Chetty, 1996).

Los pasos para seguir fueron determinar las preguntas de investigación, selección de las proposiciones teó- 
ricas, determinar la unidad de análisis, realizar la vinculación lógica de los datos a las proposiciones y definir los criterios para la interpretación de los datos.

\section{Resultados}

\section{Definición de clúster desde el enfoque de teoría de la complejidad}

En este documento se busca consolidar la visión de clúster como un sistema complejo impulsado por su dinámica interna de ciclos auto sostenidos de transacciones sociales. La dinámica del clúster esta basada en mecanismos positivos de retroalimentación auto sostenida y en la capacidad de red interconectada de relaciones dentro de un entorno definido que parte de la organización macro agregada y por un conjunto de trayectorias tecnológicas interrelacionadas. Así mismo, por mecanismos de retroalimentación y redes de relaciones. Estas redes de relaciones son la fuente de complesificación del clúster, como resultado de un aumento en densidad y frecuencia de las transacciones sociales entre agentes (Boisot, 1998). En el caso del BIT Center, viene de una dinámica del sector de las Tecnologías de Información, impulsado por la Cámara Nacional de la Industria Electrónica de Telecomunicaciones y Tecnologías de la Información (CANIETI) y con el apoyo del Consejo Nacional de Ciencia y Tecnología (CONACYT), del Gobierno del Estado, y la Universidad Autónoma de Baja California (UABC). Este proyecto busca ser un albergue a empresas, asociaciones y centros académicos y de investigación relacionadas con la industria de las TIC's que operan en Baja California. Esta concentración en un mismo espacio físico, les permitiría compartir conocimientos, experiencias, costos, imagen, servicios e infraestructura, además de crear sinergias positivas para los participantes.

\section{Fase de surgimiento de la organización clúster}

Lo primero que se observa en cuanto al surgimiento de la iniciativa de clúster es la creación de una red de agentes interactivos. Las transacciones repetidas entre agentes generan un patrón de relaciones que define la conectividad de la red. Estas transacciones pueden tener lugar verticalmente a lo largo de la cadena de valor de la industria, u horizontalmente entre competidores, empresas 0 agentes no relacionados y puede tomar forma de interdependencias negociadas o no negociadas. En una red genérica, como una red booleana, definida por un tipo de nodo y un tipo de enlace, cuando el número de enlaces se vuelve comparable al número de nodos, entonces surge una red gigante (Kauffman, 1995). La dinámica del sistema se vuelve dominado por los efectos generados por las múltiples interdependencias entre nodos. Bajo esta condición, el comportamiento del sistema experimenta una transición de fase desde linealidad a no linealidad (característica 4) y de evolución a coevolución (característica 10). El comportamiento del sistema no puede derivarse de la suma de sus partes individuales, sino que debe tener en cuenta a los primeros fenómenos cooperativos que surgen de la retroalimentación múltiple, y por otro lado, la distinción entre propiedades jerárquicas dependientes de la escala.

Los agentes son las unidades básicas de un sistema complejo. Si los agentes se definen como cualquier actor capaz para realizar una transacción social, las personas son el primer tipo de agentes, pero no el único. Es importante incluir tanto personas, como empresas e instituciones que también pueden realizar transacciones. Es importante mostrar lo importante de los agentes legales, pues suele pasarse por alto dos hechos: el primero es que la mayoría de las transacciones entre agentes legales tienen lugar fuera de los canales formales de transacciones establecidas por los agentes legales. En segundo lugar, que algunas de las transacciones mas importantes, como las relacionadas con la creación de conocimiento (innovación) o de nuevas actividades emprendedoras, como start-ups o spin-offs, son realizadas por individuos o comunidades que pueden o no ser influenciadas por estructuras legales. Stacey en 1995, describe el papel de agrupaciones espontáneas dentro de la organización que juegan en la innovación radical, afirmando que la naturaleza caótica de las innovaciones radicales requiere de comunidades mas en sintonía con la complejidad de la innovación.

Otro ejemplo que se encuentra fuera de las estructuras legales de la organización esta documentada por Becattini en 1997 y Malone y Laubacher en 1998, donde hablan de la figura del Impannatore, que es un clúster industrial textil de Prato, Italia. Los Impannatori, son arquitectos empresariales. Su función es analizar el contexto, identificar nuevos mercados, idear un nuevo producto (colección de moda) y organizar, en torno a él. Con esto se crea un consorcio de proveedores de vida limitada (Hall, 1999).

En el caso del BIT Center se tiene que uno de los principales objetivos de organizarse es de tener acceso a recursos públicos que ayuden a la realización de proyectos de innovación. Si bien es cierto existen programas que incentivan a la cooperación entre empresa y una o varias instituciones de educación superior. El lograr formalizar proyectos dentro de la industria tiene un impacto en el desarrollo de esta, pues los resultados del proyecto no únicamente quedan en la empresa participante, sino que se transfieren a otras empresas que por ser parte del clúster tienen acceso por lo menos a la socialización de los resultados. Dicha socialización es el proceso reiterativo de retroalimentación, que sirve para 
ir involucrando a nuevos actores a que participen en los nuevos proyectos. Por otro lado, la misma necesidad de generar innovaciones va creando una idea clara de tendencias que se van formando en el mercado, lo cual va propiciando un caldo de cultivo para la generación de nuevos emprendimientos. Así mismo, los participantes de los proyectos van desarrollando nuevas capacidades y habilidades que les permiten emprender. Por otro lado, las instituciones de educación superior participantes van creando spin offs gracias a las experiencias de participar en dichos proyectos.

Un vínculo en un entorno socioeconómico toma la forma de una transacción social. Dos preguntas que son relevantes sobre las transacciones sociales y la fase de transición son: a) ¿en qué condiciones se establece una red de transacciones sociales?, y b) ¿cuáles son los mecanismos que favorecen un aumento en la intensidad de las transacciones? En la literatura sobre clústeres industriales se señala que las economías flexibles dependen de una alta confianza de relaciones que se refuerzan a través de sus operaciones pero que no pueden generarse por si mismos (Sabel, 1989). Pero, según Axelrod (1984) y la teoría de juegos, la preexistencia de confianza no es una condición previa para el inicio del comportamiento cooperativo. Sino que, la cooperación puede prosperar en un mundo donde existen agentes egoístas, si es probable que las transacciones se repitan. La condición para la iteración se proporciona en un grupo geográfico por el factor de proximidad. De hecho, si los agentes saben que es probable que las transacciones se repitan, entonces es probable que eventuales comportamientos oportunistas sean correspondidos y por otro lado, si se conoce la identidad de los agentes, es menos probable que la deserción represente una estrategia de éxito. Por lo tanto, cuanto mayor sea el grado de correlación (densidad y frecuencia de transacciones), mayor es la probabilidad de que surja un comportamiento cooperativo. El surgimiento de la cooperación provoca la aparición de un sistema de retroalimentación interna dentro de la red, por lo cual la acción de un solo agente afecta directa o indirectamente la aptitud de los otros agentes, determinando así la formación de unas comunidades de agentes, obedeciendo reglas comunes y utilizando conjuntos comunes de valores y creencias. Dicho de otra forma, el bloque de la red en un patrón duradero de interdependencias genera el surgimiento de reglas compartidas de comportamiento y mentalidad. Cuando esto ocurre, la red se caracteriza por una estructura de orden superior (característica 11), capaz de reacción colectiva y comportamiento anticipativo.

EI BIT Center surge como iniciativa de agentes económicos, sin embargo, tiene una fuerte relación el vinculo que estos agentes económicos tienen con otras experiencias las que incentivaron a la formalización y búsqueda de la consolidación del BIT Center. Experien- cias previas a nivel nacional e internacional que le dan forma a la iniciativa.

La intensidad de las transacciones es un factor clave para el surgimiento de esa red de relaciones que define la especificidad de un clúster: la capacidad de los agentes económicos de coordinar sus micro actividades en un patrón coherente. La intensidad de las transacciones también es clave para la aparición de no linealidades en el sistema. Si la producción de un bien o servicio requiere un conjunto complejo de transacciones no sujetas al control externo, la coordinación debe estar asegurada por los múltiples bucles de retroalimentación que unen a los agentes en una red.

El aumento de la intensidad de las transacciones como fenómenos que tienen lugar durante un tiempo y en un territorio delimitado geográficamente puede leerse como un efecto de aglomeración. Como Fujita et al., en 1999 señalan: el problema básico de hacer teoría geográfica económica ha sido siempre la observación de que cualquier historia sobre la región y el desarrollo urbano debe depender de manera crucial del papel de los rendimientos crecientes. Los rendimientos crecientes y la intensidad de las transacciones son similares al concepto de reacción auto catalítica presentada en la característica 12. Este punto, demuestra que se necesita alguna forma de masa crítica para describir el inicio de la espiral de rendimientos crecientes o reacción auto catalítica. Es bien sabido, que cuando el comportamiento de los actores económicos se vuelve interdependiente con lo que hacen otros agentes, los modelos de masa crítica se vuelven importantes. El concepto de masa crítica, aglomeración como manifestación de rendimientos crecientes y auto catalíticos sugieren que la evolución de una red de agentes económicos puede generar un clúster de rendimientos crecientes cuando la intensidad de las transacciones dentro del clúster alcanza un valor crítico. Se caracteriza una red de organizaciones que atraviesa una fase de transición por las siguientes propiedades: a) desplazamiento de las actividades de creación de valor fuera de los nodos de la red, b) aparición de una espiral entre las fuerzas de la diversidad y los efectos del reciclaje y multiplicador, c) aparición de un lugar complejo, y d) aparición de un sistema distribuido de conocimiento.

El BIT Center tiene la idea de que gracias a la proximidad de las empresas dentro del inmueble BIT Center, facilitara las relaciones entre los miembros del clúster, lo que significa un aumento de la intensidad y la cantidad de relaciones entre miembros. En estas relaciones es donde esta el valor del clúster, pues es como se van revelando las preferencias de los agentes, así como la entropía del sistema se disminuye por el flujo de información. Además, la ubicación del inmueble permite tener acceso a otras industrias del mismo sector, las cuales aún sin pertenecer al clúster pueden integrarse en 
algunas actividades. Por otro lado, las relaciones entre los miembros del clúster se pueden identificar complementariedades que en un momento dado pueden servir para atender a nuevos mercados, o crear mercados, mediante la diversificación de productos, incluso como consecuencia de la especialización.

El valor creado por un grupo de empresas que interactúan libremente entre sí (red), es la suma de los valores generados en cada nodo individual (dinámica lineal). En el caso de las empresas agrupadas, el valor total es mayor que la suma de los valores generados en cada nodo (dinámica no lineal), por que una parte relevante del proceso de creación de valor tiene lugar a nivel interfaz. Debido al efecto de la espiral, el entorno de transacciones sociales que solía ser mayoritariamente interno a la empresa ha sido sustituido por el sistema de relaciones entre los agentes especializados del clúster. Dicho de otra forma, un clúster representa una forma de gobernanza en la que el proceso de creación de valor se transfiere al sistema de relaciones en el clúster. Dependiendo el enfoque, el supuesto de que la empresa representa la unidad de análisis de los estudios económicos y organizativos, se vuelve cuestionable (Arthur y Rousseau, 1996). La unidad de análisis se convierte en el sistema de relaciones entre organizaciones, no necesariamente empresas, y el proceso económico se convierte en un proceso de conversación y coordinación entre agentes.

En el caso del BIT Center, su estudio como clúster va mas allá de la simple suma de sus inquilinos, sino que en su creación tiene una junta directiva que toma decisiones estratégicas en beneficio del desarrollo del sector y de la comunidad en general al fomentar el uso de las TIC's. Sin embargo, debe existir una planeación estratégica acorde a las características de la literatura de los objetivos de un clúster, pues no es suficiente con llevar la parte de la dirección y de la una junta directiva, sino que es necesario trabajar en la planeación estratégica y definir el rumbo del clúster, además de buscas formas de generar relaciones de forma óptima.

La fragmentación, provocada por los procesos de verticalidad, desintegración y especialización flexible, junto con la diferenciación interna generado por la internalización de la oferta y demanda dentro de los límites del clúster (Porter, 1998), hace que la variedad interna de los agentes incremente. El número y complejidad de nichos de mercado dentro del área del clúster aumenta y la propiedad de los productos finales se traslada de los nodos al interior del clúster.

La variedad desencadena un fenómeno de efecto multiplicador. Es conocido en economía de que una transacción puede tener un efecto en cascada sobre transacciones sucesivas. El efecto multiplicador juega un papel importante en los sistemas jerárquicos o de mercado tradicionales. Este efecto multiplicador captu- ra el hecho de que a medida que crece el tamaño de la economía del clúster, se vuelve más conveniente para producir una distribución más amplia de productos y servicios dentro del clúster, dando lugar a una dinámica de rendimientos crecientes.

El mismo recurso, ya sea tangible o intangible, se reutiliza dentro del clúster, con mas intensidad que en el caso de una organización aislada. John Holland, 1995, comenta que el reciclaje puede aumentar la producción, pero el efecto general del reciclaje dentro de una red con muchos ciclos puede ser explosiva. Holland ejemplifica con una selva tropical y comenta que esta es de un suelo extremadamente pobre, sin embargo, el bosque en si es rico tanto en especies como en su cantidad. Esta situación depende casi por completo de la capacidad del bosque para capturar y reciclar recursos críticos para el ecosistema.

Por otro lado, el crecimiento dentro de un clúster se lleva a cabo mediante la reducción de costos, o por innovaciones incrementales o radicales. En cuanto a los costos, la competencia se describe como competencia perfecta de mercado y, en términos biológicos, está regulada por la ley darwiniana de selección. Este es un proceso que no afecta y que no depende de el grado de variedad del sistema. La competencia esta impulsada por la creación de nuevos nichos de mercado o por nueva tecnología y se define como competencia shumpeteriana. Este proceso es similar al proceso de especialización en ecología y es un proceso generador de variedad, porque las especies pertenecientes a diferentes nichos no competirán directamente entre si. El equilibro entre los dos efectos (mercado perfecto frente al shumpeteriano o selectivo, presión versus especialización) depende de la variedad del sistema y de la tasa de cambio de variedad. Cuanto mayor sea el grado de variedad del sistema y la velocidad de cambio sea más rápida, la competencia shumpeteriana más dominante será, según Gibbons y Metcalfer (1986), cuando la tasa de innovación dentro de una industria sea directamente proporcional a su grado de variedad económica. El terreno de la competencia se moverá del tipo de interacción presa / depredador con su proceso característico de muerte / reemplazo al proceso de separación de nichos basado en redes de complementariedades e innovación. El equilibro entre los dos mecanismos de competencia (Mckelvey, 1999) depende de la tasa de coevolución, que se vuelve dominante en la fase de transición, donde se logra el valor de las transacciones sociales y entra en juego una variedad de mecanismos en espiral. La distinción entre la importancia relativa del mercado shumpeteriano frente al mercado perfecto la competencia puede ayudar a trazar otro elemento de distinción entre clúster y redes.

Una de las principales ideas de impulsar la creación del BIT Center es la especialización del sector, la cuál no 
surge espontáneamente, sino que es resultado de las interacciones entre los actores de un mercado. También pueden surgir ideas sobre oportunidades de especialización como resultado de un estudio de mercado y enfocarse a lograr nuevas competencias para ofrecer nuevos productos o servicios. Por otro lado, también es importante estudiar la dinámica que se esta dando en los actores miembros y no miembros del clúster, pues estos de alguna forma están revelando que tendencias están viendo. Por último, la especialización de una industria también puede venir de la suma de dos industrias, y que en conjunto creen una nueva, o que transformen una industria tradicional en una mas tecnificada.

\section{Aparición de un lugar complejo}

Cuando un conjunto de organizaciones se convierte en una red autosuficiente de transacciones interdependientes, el sistema puede sufrir una transición de fase donde surgen propiedades sistémicas, sin embargo, estas propiedades no necesariamente representan el surgimiento de un nivel superior de organización. El surgimiento del nivel superior de organización constituye un cambio ambiental. La complejidad que aporta la variedad de generadores de mecanismos y que estos son posibilitados por la propiedad de proximidad, lo cual produce un nuevo entorno. Con la diferenciación entre el entorno interno del clúster, externo a la organización, pero interno al clúster, y el externo (externo tanto a las organizaciones como al clúster). Los dos entornos son diferentes en términos de tipo de dinámica y procesos de creación de valor. Los sistemas complejos se pueden diferenciar de los no complejos por su capacidad de generar su propio lugar, es decir, anidar dentro del entorno externo un tipo específico de lugar, hecho por una mezcla única de cultura territorial, tecnología y formas organizativas. Dicho lugar se define, por un alto grado de acoplamiento entre el lugar y los agentes; por una comunidad de conocimientos y prácticas; y por una trayectoria tecnológica envolvente (Dosi \& Orsenigo, 1985).

El acoplamiento de los agentes, a diferencia de un entorno tradicional, donde los agentes actúan de forma estática (desde el punto de vista de los agentes), existe un fuerte acoplamiento coevolutivo entre agentes y grupo. Este entorno cercano es el resultado de la conectividad de los agentes y por lo tanto sensible a las acciones de los agentes. Las estrategias de planeación estratégica, que prosperan en un entorno estático con un grado bajo, o nulo, de acoplamiento entre los agentes, y medio ambiente, son un ejemplo de estrategia darwiniana o evolutiva. En cambio, las estrategias coevolutivas son necesarias cuando hay un acoplamiento significativo y necesitan tener en cuenta los efectos de red no lineales (Arthur, 1996).

El lugar creado por la interacción compleja de los agentes coincide con un Lamarckiano, es decir, una comunidad definida por una mezcla territorialmente delimitada de competitividad / y comportamiento de cooperación. La unidad de análisis socioeconómico cambia de la organización a lo que define el actor socioeconómico de la comunidad. La comunidad se basa en un conjunto de valores locales y un enfoque específico para el aprendizaje y el intercambio de información, que es en gran parte inconsciente e inadvertido (Becattini, 1998). También se llevan a cabo formas similares de intercambio de información fuera de la comunidad del clúster, pero sin la misma ética de libre comunicación, ni prácticas de intercambio y cooperación y ni a la misma escala. La actitud frente al intercambio de información con la competencia es la verdadera prueba de fuego. Este tipo de intercambio de información es menos sorprendente si se piensa que en un clúster sería difícil ocultar una determinada información. Pues puede ser conveniente cambiarla por una información en el futuro.

El BIT Center tiene unos nobles ideales de cooperación para el desarrollo y fomento del sector en la región, las relaciones que se den entre los agentes involucrados en el clúster son los que marcaran el ritmo del desarrollo. Desde la óptica de la teoría de la complejidad se sabe que entre mayor sean las interacciones mas se intensifica la comunicación y por ende se generan nuevas opciones de resultados. Es importante que dentro del clúster se fomente la integración y cooperación de los miembros en diferentes actividades, desde actividades rompehielo hasta el desarrollo de eventos en conjunto y así hasta llegar a la elaboración de proyectos. La confianza es algo que se tiene que ir construyendo y es de poco a poco, pero lo importante es iniciar. De ahí lo importante de iniciar el ejercicio de prueba y error en las relaciones e irlas fortaleciendo poco a poco, incluso desde un ámbito informal.

Un clúster esta formado por un conjunto de organizaciones unidos por relaciones de complementariedad, proximidad e historia. Esta densa e intensa red de complementariedades entre usuarios y productores de la tecnología, dentro y a través de diferentes sectores define un lugar tecnológico que se convierte con el tiempo en endogeneizado. Los límites de este lugar endógeno están definidos por el conjunto de trayectorias tecnológicas interrelacionadas (Dosi y Orsenigo, 1985) que surgen a través del proceso espontáneo y auto organizado de un enfoque de ensayo y error. Este lugar marca una diferencia de la red (cadena de suministro) o tipo de organización independiente debido al proceso centralizado de coordinación. Las opciones tecnológicas tienden a estar restringido en torno a una sola tecnología o trayectoria tecnológica.

El clúster como un sistema distribuido de conocimiento, se ve como una forma de meta organización, 
pues sus elementos pueden auto organizarse y generar un nuevo nivel organizacional que está definido por un lugar adecuado y un conjunto específico de valores y prácticas. Este nivel organizacional debe ser autónomamente capaz, con el fin de percibir sus cambios internos e interactuar con los externos para: a) recopilar datos, b) organizarlos en patrones informativos, c) destilar una representación operativa (conocimiento), y d) almacenar esa información para uso futuro.

Ante el planteamiento de como los sistemas puramente descentralizados pueden procesar información de forma orgánica, el problema de representación en un grupo se puede explicar parcialmente por los mecanismos de una representación de una red neuronal: una red neuronal consta de un gran número de neuronas simples que se encuentran interconectadas. Los pesos asociados con las conexiones entre neuronas determinan las características de la red. Durante un período de entrenamiento, la red ajusta los valores de la interconexión. El valor de cualquier peso específico no tiene importancia, es el patrón de valores de ponderaciones en todo el sistema que contienen información. Estos patrones son complejos y son generados por la propia red, no hay un procedimiento abstracto disponible para describir el proceso utilizado por la red para resolver el problema (Cilliers, 1998).

Como una red neuronal, la información se transforma en conocimiento y luego en acción, sin el prerrequisito de un sistema semántico de reglas. No hay necesidad de un sistema centralizado que almacena información y dicta las reglas de comportamiento. La tradicional vista piramidal de las empresas como una jerarquía organizada que recopila y transmite datos e información de abajo hacia arriba. El conocimiento se codifica en la parte superior y crea una sintaxis que luego se filtrara hacia abajo, la organización no se mantiene en un grupo. La memoria simplemente se almacena en los patrones de conexiones entre agentes distribuidos y en los vínculos intra e interorganizacionales. La creación de conocimiento implica un cambio en el patrón de conexiones, una reorganización de la geografía y tipología de enlaces. El punto crucial es que esta reorganización no se basa en una estrategia deliberada, sino por el contrario, es una consecuencia involuntaria producto de los intentos de los agentes de maximizar su aptitud mediante interacciones e información locales.

\section{Conclusiones}

La literatura sobre clúster ha hecho uso de una variedad de marcos, todos centrados en el concepto de economía y sociología de la forma de organización en red, este documento sugiere el uso de la teoría de la complejidad, pues esta puede contribuir en la reinterpretación el fenómeno de la aglomeración industrial a la luz de una teoría dinámica y relacional basada en el poder de las relaciones no lineales entre los agentes. Además, se basa en el concepto de emergencia y auto organización y sobre el resultado de la investigación sobre clúster. Este documento presenta las siguientes ideas: a) la forma organizativa de un clúster es una propiedad emergente del recurrente patrón de transacciones sociales que tienen lugar en una red de organizaciones que interactúan sobre un territorio geográfico; b) el clúster se define por la creación de un tipo especifico de entorno basado en un conjunto de trayectorias tecnologías y productivas, por un sistema distribuido de conocimiento y por una comunidad.

El clúster internaliza los aspectos socioeconómicos y relacionales fundamentales, como usuarios-productores mediante la coincidencia territorial de oferta y la demanda, y reduce la incertidumbre a un nivel manejable a través de los mencionados internalización y exploración de un conjunto de trayectorias tecnológicas interrelacionadas.

Los clústeres son impulsados por una dinámica interna de retroalimentación positiva auto sostenida, ciclos de transacciones sociales. Estos ciclos se vuelven autosuficientes cuando se logra una densidad crítica de transacciones sociales. Bajo esta condición en fase de transición determina la evolución de una red a un clúster.

El clúster depende para su supervivencia de la innovación continua y representa una forma adaptativa a las fluctuaciones extremas del mercado. Estandarización de técnicas y ubiquitinación de conocimientos son incompatibles en el clúster. 


\section{Referencias bibliográficas}

Arthur, W. B. (1996). Increasing returns and the new world of business. Harvard Business Review, July-August.

Axelrod, R. (1984). The evolution of co-operation. London: New Basic Books.

Bak, P., \& Chen, K. (1991). Self-organised criticality. Scientific American, January.

Basant, R. (2006). "Bangalore cluster: Evolution, Growth and Challenges". Working Paper 2006-05-02. Indian Institute of Management. Ahmedabad - 380015.

Becattini, G. (1990). "The Marshallian industrial district as a socio-economic notion". In F. Pyke, G. Becattini y W. Sengenberger (Eds.). Industrial districts and interfirm co-operation in Italy. Geneva: International Institute for Labour Studies.

Becattini, G. (1997). Prato: Storia di una Citta ': il distretto industriale. Florencia: Le Monnier.

Becattini, G. (1998). Distretti industriali e made in Italy. Torino: Bollati Boringhieri.

Boisot, M. (1998). Knowledge assets: Securing competitive advantage in the information economy. Oxford: Oxford University Press.

Chetty S. (1996). The case study method for research in small- and médium - sized firms. International small business journal, vol. 5, octubre - diciembre.

Cilliers, P. (1998). Complexity and Postmodernism. Londres: Routledge.

Dosi, G., \& Orsenigo, L. (1985). Order and change: An exploration of markets, institutions and technology in industrial dynamics. Brighton: SPRU, discussion paper, No. 32.

Florida, R. (2005). "Cities and the creative class". Abingdon: Routledge.

Fujita, M., Venables, A., \& Krugman, P. (1999). The spatial economy: Cities, regions and international trade. Cambridge, Mass: MIT Press.

Gibbons, M., \& Metcalfe, J. (1986). Technology, variety and competition. In: I. Prigogine, \& M. Sanglier (Eds), Laws of nature and human conduct. Brussels: Academie Royal Belgique.

Hall, R. (1999). Rearranging risks and rewards in a supply chain. Journal of General Management, 4 (3), Spring.

Holland, J. H. (1995). Hidden order: How adaptation builds complexity. Massachusetts: Addison Wesley.

Jacobs, J. (1969). "Economy of cities". New York: Vintage.

Kauffman, S. (1995). At home in the universe. Oxford: Prensa de la Universidad de Oxford.

Krugman, P. (1995). "Development, geography and economic theory". Cambridge, MA: The MIT Press.

Lindqvist, G. (2009). "Disentangling clusters: agglomer- ation and proximity effects". Disertasión PhD tesis en Stockholm School of Economics. Jönköping University, 2009.

Malmberg, A., Sölvell, Ö., \& Zander, I. (1996). "Spatial clustering, local accumulation of kknowledge and firm competitiveness". Geografisca annaler, 78 B(2), 85-97

Marshall, A. (1920). "Principles of Economics". 8th ed. Vol. Libro IV. London: Macmillan.

Malone, T. W., \& Laubacher, R. J. (1998). The dawn of the E-lance economy. Harvard Business Review, September-October, 149 ..

McKelvey, B. (1999). Visionary leadership vs. distributed intelligence: Strategy, micro-evolution, complexity. EIASM Workshop on Complexity and Organisation. Brussels, June.

Nicolis, G., \& Prigogine, I. (1989). Exploring complexity: An introduction. New York: Freeman.

Porter, M. (1998). Clusters and the new economics of competition. Harvard Business Review, November-December

Norberg, A. (1992). "The origins of the Electronics Industry on the Pacific Coast". Proceedings of the Institute of Electrical and Electronics Engineers 64. Num. 9. pp 1314 - 1322.

Piore, M., \& Sabel, C. (1984). "The second industrial divide: possibilities for prosperity". New York, NY: Basic Books.

Pyke, F., Becattini, G., \& Sengenberger, W. (1990). "Industrial districts and inter-firm co-operation in Italy". Geneva: International Institute for Labour Studies.

Ramírez, B., Osuna, J. \& Suástegui, B. (2012). "Vinculacion Uniersidad - Sociedad y el modelo de la Triple Hélice". XIII Asamblea General de ALAFEC. Asociación Latinoamericana de Facultades y Escuelas de Contaduria y Administración. Obtenido en: http://132.248.164.227/alafec/docs/asambleas/xiii/ ponencias/educacion/pdf/E_06.pdf

Rosegrant, S. \& Lampe, D. (1992). "Route 128: Lessons from Boston 's High-Tech Community". New York: Basic Books.

Sabel, C. F. (1989). Flexible specialisation and the re-emergence of regional economies. In: P. Hirst, \& J. Zeitlin (Eds), Reversing industrial decline? Oxford: Berg.

Shelling, T. C. (1978). Micromotives and Macrobehaviour. New York: Norton \& Company

Stacey, R. D. (1995). The science of complexity: An alternative perspective for strategic change processes. Strategic Management Journal, 16 ..

Waldrop, M. M. (1992). Complexity, the emerging science at the edge of order and chaos. London: Penguin. 